\title{
Diversidad y abundancia relativa de corales, octocorales y esponjas en el Parque Nacional Jaragua, República Dominicana
}

\author{
Ernesto Weil \\ Department of Marine Sciences, University of Puerto Rico. PO BOX 908, Lajas, PR 00667; eweil@caribe.net
}

Recibido 19-X-2001. Corregido 15-I-2004. Aceptado 07-IX-2004.

\begin{abstract}
Diversity and relative abundance of corals, octocorals and sponges at Jaragua National Park, Dominican Republic. The Jaragua National Park is located in a remote area to the SW coast of the Dominican Republic. Fishing and mining are the major human activities. The main reef formations of the Park include: (a) long bank reefs (spur and groove) growing as bands over the platform and running in a SW-NW direction at 12-25 m depth, (b) well developed, deep, fringing reefs at the platform edge (drop-off) areas which could extend from 10 to $45 \mathrm{~m}$ depth, and (c) small patch reefs and poorly developed coral-octocoral-sponge-algal communities in shallow platforms near shore, rocky bottoms, and over the submerged walls of the uplifted reef. Nine reef localities were surveyed between Cabo Beata and Bahia Honda using Scuba diving to inventory the diversity and relative abundance of scleractinian corals, octocorals and sponges. Fringing reefs were surveyed starting at the bottom $(30 \mathrm{~m})$ and swimming in a zig-zag pattern $(50 \mathrm{~m}$ on each side) to shallower areas. Bank reefs were surveyed by swimming in zig-zag across the spur-groove formation along $500 \mathrm{~m}$. Sponges were the most diverse group with 83 species in 50 genera followed by the scleractinian corals with 56 species in 26 genera and the octocorals with 47 species in 15 genera. New records included eight coral species, 29 octocoral species and 59 sponges. The diversity, species composition and abundance of particular groups varied across the different localities. Northern reefs within the park and the Los Frailes Island offshore had the highest live cover, relative abundance and diversity for the three groups. In general, the Jaragua National Park had the highest diversity of corals, octocorals and sponges reported for the Dominican Republic and rank amongst the highest reported for the northern Caribbean. It is recommended that the area be protected and that fishing activities be regulated or eliminated altogether. Rev. Biol. Trop. 54(2): 423-443. Epub 2006 Jun 01.
\end{abstract}

Key words: diversity, relative abundance, coral reefs, coral, octocorals, sponges, Jaragua National Park, Dominican Republic, Caribbean.

Los estudios cuantitativos de diversidad de corales escleractínidos en la República Dominicana comienzan con el inventario de Bonnelly de Calventi (1974). Este inventario se limita a una profundidad de $9 \mathrm{~m}$ y compila 27 especies con zooxantelas y una especie sin zooxantelas para varias localidades. Geraldes y Bonnelly de Calventi (1978) completan este primer listado con inventarios hechos en los arrecifes de la costa Sur hasta una profundidad de $30 \mathrm{~m}$. Estos autores registraron 43 especies con zooxantelas y a Madracis pharensis como la única especie sin zooxantelas observada. Posteriormente, un inventario exhaustivo de las plantas y animales marinos en tres localidades de la costa SE hasta $20 \mathrm{~m}$ de profundidad produjo una lista de 40 especies corales, todas con zooxantelas y ninguna de las cuales constituye un registro nuevo para la isla (Williams et al. 1983). En 1994 se realizó el estudio de mayor extensión geográfica hasta la fecha, muestreando 14 localidades a lo largo de la costa dominicana (Geraldes 1994). Este autor identificó 36 especies con zooxantelas para estas localidades sin ningún registro nuevo para la República Dominicana.

Los inventarios de otros grupos de cnidarios y esponjas para las áreas arrecifales 
han sido aun más limitados. Bardales (fide Williams et al. 1983) registra 24 especies de esponjas pertenecientes a 18 géneros y 26 especies de octocorales pertenecientes a 11 géneros para tres localidades del SE dominicano. El esfuerzo más reciente fue una evaluación de la biodiversidad marina de la República Dominicana con comentarios sobre la distribución de organismos, habitáculos y el estado actual (Ottenwalder et al. 1999).

El objetivo principal de este estudio fue caracterizar cualitativamente las comunidades coralinas del Parque Nacional Jaragua en la costa sur-occidental de la República Dominicana. El estudio se realizó en 1997 durante una expedición del Programa de Estudio de la Diversidad Marino-Costera de la República Dominicana. Nueve localidades representativas del Parque fueron seleccionadas y muestreadas para producir un inventario lo más completo posible de la riqueza de especies de corales escleractínidos, octocorales y esponjas. Además se estimaron cualitativamente las abundancias relativas de las especies más comunes. Este trabajo, junto con el informe inicial de los resultados (Weil 1997), representan los primeros inventarios comprehensivos de las especies de corales escleractínidos, octocorales y esponjas para el extremo sur occidental de la República Dominicana.

\section{MATERIALES Y MÉTODOS}

Área de estudio. El Parque Nacional Jaragua está localizado en la costa sur occidental de la República Dominicana (Fig. 1). El área se caracteriza por un clima árido y una vegetación desértica sobre unas terrazas arrecifales emergidas (Pleistoceno) sin ríos o fuentes de agua superficiales (Geraldes 2003). El Parque tiene una extensión de costa de unos $125 \mathrm{~km}$ y un área aproximada de arrecifes de $4.6 \mathrm{~km}^{2}$. Incluye una variedad de comunidades terrestres y marinas que se encuentran alejadas de áreas densamente pobladas y por lo tanto, de impactos antropogénicos directos e intensos. Las actividades humanas más importantes en el área son la pesca y la antigua mina de bauxita en Cabo Rojo. El área y los muelles son actualmente usados como puerto de carga de gravilla para construcción.

Las zonas marino-costeras están dominadas por bosques de manglar, playas arenosas y riscos (paredes verticales) de arrecifes emergidos. Las áreas someras generalmente están dominadas por praderas de fanerógamas marinas, principalmente Thalassia testudinum y Siringodium filiforme, fondos arenosos, fondos rocosos y/o paredes con comunidades bentónicas dominadas principalmente por especies de corales creciendo en forma incrustante (Porites astreoides, Montastraea faveolata, Diploria clivosa, Colpophyllia natans, etc.), una alta variedad de esponjas, octocorales, hidrozoarios, zoántidos coloniales, y algas. Las principales formaciones arrecifales del área son los arrecifes de banco ó montículo. Estas estructuras alargadas se desarrollan sobre la plataforma insular y se extienden como bandas

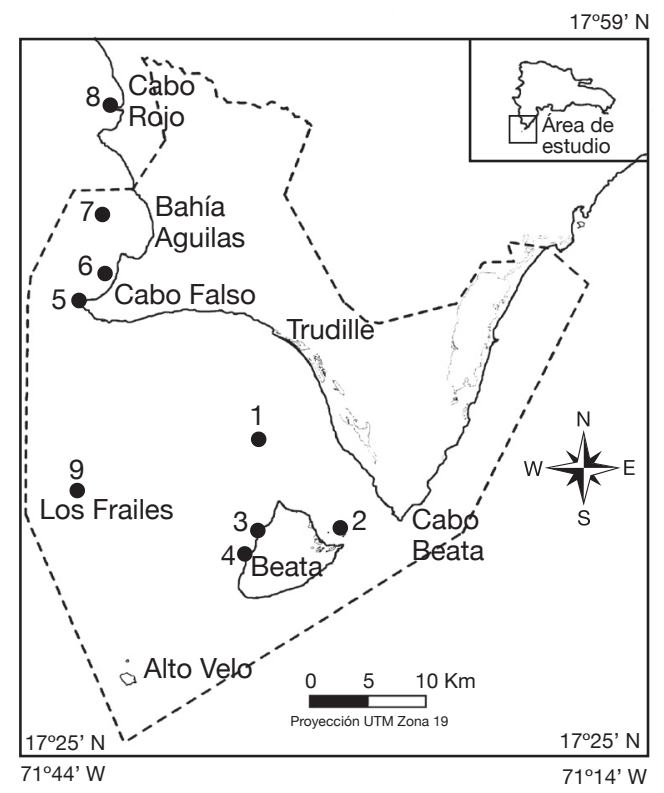

Fig. 1. Mapa del sur-oeste de la República Dominicana mostrando el área del Parque Nacional Jaragua y las localidades de estudio. 1= Grumelandia; 2= Los Carraplanes; 3= Playa del Coco; 4= Punta Lanza; 5= Cabo Falso; 6= Lanza Zo; 7= Bahia de Aguilas; 9= Bahia Honda; 9= Los Frailes. 
paralelas por cientos de metros en dirección SE - NW a distancias variables de la costa. Estos arrecifes se encuentran en zonas de profundidad intermedia (10-20 m) y varían en tamaño, forma y longitud. En los bordes de la plataforma se desarrollan arrecifes de franja extensos y muy diversos, con una alta cobertura viva de corales, otros cnidarios y esponjas. En la plataforma somera y áreas cercanas a la costa, se encuentran arrecifes de parche pequeños y comunidades coralinas extensas con colonias incrustantes y dispersas de corales, octocorales, hidrocorales, zoántidos y esponjas, y una alta cobertura de algas. Estas comunidades se distribuyen en las paredes verticales de los riscos, los fondos rocosos y los sustratos duros a lo largo de todo el Parque.

Ocho localidades de estudio distribuidas en el área occidental del Parque Jaragua (Fig. 1; Cuadro 1) fueron seleccionadas usando información fotográfica y cartas marinas. Estas localidades representan la variedad de formaciones arrecifales y comunidades coralinas presentes en el área. Dentro del Parque, cinco de estas localidades están cercanas a la costa y a Isla Beata. Dos son arrecifes de banco largos sobre la plataforma en el canal de Beata y al oeste de Bahía de Aguilas y la última fue el arrecife de franja de la isla Los Frailes, a unos $20 \mathrm{~km}$ mar afuera hacia el oeste. La novena localidad fue un arrecife de franja profundo muy bien desarrollado y diverso localizado en Bahía Honda, Cabo Rojo. El arrecife bordea el norte del canal de entrada de embarcaciones al frente de la mina de bauxita, cerca del norte de los linderos de Parque Jaragua. El tope del arrecife está a 10-12 $\mathrm{m}$ de profundidad y se extiende hasta $45 \mathrm{~m}$ de profundidad en algunas áreas. La zona sur-oriental del Parque, al este de Cabo Beata, no se pudo muestrear debido a las malas condiciones del tiempo.

Metodología. Este estudio se realizó durante la segunda expedición exploratoria al Parque Nacional Jaragua del Programa de Diversidad Marino-Costera en el verano de 1997. En cada una de las nueve localidades (Cuadro 1) se realizó un censo intensivo (por lo menos dos buceos) de las especies de corales, octocorales y esponjas y una estimación cualitativa de las abundancias relativas de cada especie. Cada buceo duró entre una hora a una hora y media. En los arrecifes de banco se nadaba en zig-zag (cubriendo el ancho de la estructura arrecifal) a lo largo del arrecife por una distancia de aproximadamente $500 \mathrm{~m}$. En

CUADRO 1

Tipo, profundidad y ubicación geográfica de los arrecifes estudiados

\begin{tabular}{llccc}
\multicolumn{1}{c}{ Arrecife } & \multicolumn{1}{c}{ Tipo de arrecife } & Profundidad $(\mathrm{m})$ & Latitud & Longitud \\
GRUM & Banco & $18-25$ & $17^{\circ} 40.000$ & $71^{\circ} 32.348$ \\
PCOC & Franjeante & $8-20$ & $17^{\circ} 34.569$ & $71^{\circ} 32.950$ \\
CARRA & Franjeante & $0-10$ & $17^{\circ} 35.899$ & $71^{\circ} 28.286$ \\
PLAN & Comunidad & $0-8$ & $17^{\circ} 35.720$ & $71^{\circ} 32.330$ \\
CFAL & Franjeante & $0-18$ & $17^{\circ} 46.400$ & $71^{\circ} 41.260$ \\
LZO & Banco & $15-25$ & $17^{\circ} 47.650$ & $71^{\circ} 40.007$ \\
BAGUI & Franjeante & $15-40$ & $17^{\circ} 48.255$ & $71^{\circ} 38.630$ \\
BHONDA & Franjeante & $12-45$ & $17^{\circ} 55.745$ & $71^{\circ} 39.956$ \\
FRA & Comunidad & $0-40$ & $17^{\circ} 37.620$ & $71^{\circ} 41.190$
\end{tabular}

Localidades: $\mathrm{GRU}=$ Grumelandia; PCOC $=$ Playa del Coco CARRA $=$ Los Carraplanes; PLAN $=$ Punta Lanza; CFAL $=$ Cabo Falso; LZO = Lanza Zo; BAGUI = Bahía de Águilas; BHONDA = Bahía Honda en Cabo Rojo; FRAI = Los Frailes. Comunidad $=$ comunidad arrecifal densa pero $\sin$ estructura arrecifal consolidada. 
los arrecifes de franja, el censo comenzaba en la parte profunda $(30-40 \mathrm{~m})$ del arrecife, luego nadando en zig-zag (50 m a cada lado) hasta llegar a la zona somera o el tope del arrecife. Todas las especies presentes de los tres grupos en estas bandas de muestreo fueron identificadas in situ y sus abundancias relativas a lo largo del arrecife se estimaron en función de conteos rápidos que luego se clasificaron en tres categorías: raras o poco comunes (una a cinco colonias por arrecife), comunes (cinco a 15 colonias por arrecife) y abundantes (más de 15 colonias). Las especies dudosas y/o no reconocidas de octocorales y esponjas fueron dibujadas y descritas parcialmente en tablas de acrílico en el agua y luego, se corroboraba su descripción con guías de campo como Humann (1993) y/o con descripciones en referencias especializadas (Bayer 1973, Weidenmeyer 1978, Zea 1987) para su identificación final. La clasificación de corales siguió la nomenclatura de Wells (1973) e incorpora cambios recientes en la nomenclatura taxonómica de la familia Agaricidae (Stemman 1991) y descripciones recientes de especies nuevas de corales en el Caribe (Zlatarski 1990, Weil y Knowlton 1994). Luego de un análisis morfométrico multivariado de las especies fósiles y vivientes de Agaricia del Caribe, el género Undaria fue re-establecido como válido e incluye todas las especies someras (e.g. Undaria agaricites, $U$. humilis, $U$. purpurea), mientras que el género Agaricia contiene las especies profundas (e.g. Agaricia lamarcki y A. undata) (Stemman 1991).

\section{RESULTADOS}

Se identificaron un total de 56 especies (con una variabilidad de 19-50 sp. entre localidades) pertenecientes a 26 géneros de corales escleractínidos, 4 especies de hidrocorales, 47 especies (19-44 variabilidad entre localidades) en 15 géneros de octocorales, y 83 especies (11-62 variabilidad entre localidades) en 50 géneros de esponjas en las 9 localidades muestreadas (Cuadro 2; Apéndices 1 al 3). En general, las localidades del norte, desde Cabo Falso a Bahía Honda y la isla de Los Frailes mar afuera, presentaron las comunidades más ricas en especies, con mayores abundancias y coberturas altas de cnidarios y esponjas.

De las comunidades al sur de Parque, la costa oeste de Beata (Punta Lanza) presentó las diversidades más bajas de corales, octocorales y esponjas. Los arrecifes de Grumelandia (Fig. 1), Bahía de Aguilas y Bahía Honda en

CUADRO 2

Número de especies (y géneros) de corales, octocorales y esponjas en las distintas localidades estudiadas del Parque Nacional Jaragua al SO de la República Domincana

\begin{tabular}{|c|c|c|c|c|c|}
\hline \multirow[t]{2}{*}{ Arrecife } & \multicolumn{3}{|c|}{ Corales escleractínidos } & \multirow[t]{2}{*}{ Octocorales } & \multirow[t]{2}{*}{ Esponjas } \\
\hline & Con zooxantelas & Sin zooxantelas & Total & & \\
\hline GRUM & $40(18)$ & $5(5)$ & $45(23)$ & $19(11)$ & $38(26)$ \\
\hline PCOC & $34(20)$ & $3(3)$ & $37(22)$ & $31(10)$ & $42(26)$ \\
\hline CARRA & $21(18)$ & $3(3)$ & $24(14)$ & $25(10)$ & $30(24)$ \\
\hline PLAN & $16(14)$ & $3(3)$ & $19(12)$ & $24(6)$ & $11(10)$ \\
\hline CFAL & $45(26)$ & $5(5)$ & $50(23)$ & $41(15)$ & $68(38)$ \\
\hline LZO & $42(20)$ & $4(4)$ & $46(23)$ & $28(12)$ & $52(34)$ \\
\hline BAGUI & $44(21)$ & $4(4)$ & $48(24)$ & $44(15)$ & $61(36)$ \\
\hline BHONDA & $47(21)$ & $5(5)$ & $52(25)$ & $22(10)$ & $50(32)$ \\
\hline FRA & $44(19)$ & $5(5)$ & $49(23)$ & $41(15)$ & $62(40)$ \\
\hline Totales & - & - & $56(26)$ & $83(50)$ & $47(15)$ \\
\hline
\end{tabular}

Nota: El significado de las abreviaturas se presentan en la Tabla 1. 
Cabo Rojo, presentaron elevada riqueza y abundancias de corales y esponjas. Los Frailes, con una alta heterogeneidad espacial, fuertes corrientes y oleaje, presentó una alta diversidad y abundancia de corales, octocorales y esponjas formando una comunidad coralina bien desarrollada creciendo sobre fondo rocoso pero sin una estructura arrecifal consolidada.

En la costa oeste de Isla Beata, la presencia de arrecifes es limitada, siendo característico del área fondos consolidados con comunidades de algas, esponjas, octocorales y corales, de baja diversidad pero con una alta abundancia de los tres primeros grupos. Las paredes verticales sumergidas de los arrecifes fósiles elevados y consolidados, están colonizadas por algas crustosas calcáreas, corales incrustantes, zoántidos coloniales y esponjas principalmente. En Cabo Falso, el fondo cercano a estas paredes está formado por rocas muy grandes que se han desprendido de las paredes emergidas y que proporcionan una alta heterogeneidad espacial, la cual permite la convivencia de un elevado número de especies sésiles de diversos grupos. De observaron colonias grandes de Montastraea, Colpophyllia y Diploria pero sin una estructura arrecifal consolidada y bien desarrollada. Una descripción breve de las distintas localidades sigue a continuación:

Grumelandia. En el sur del Parque la plataforma insular se extiende desde la costa hasta Isla Beata. La profundidad promedio en el canal es de 18-20 $\mathrm{m}$ y los arrecifes de coral forman bandas y montículos arrecifales con canales de arena costa afuera extiendendose en dirección SE-NW por varios kilómetros hacia Cabo Falso. Grumelandia está formada por bancos arrecifales alargados de cientos de metros de longitud y unos 25-30 m de ancho, separadas por espacios de arena que varían en ancho y profundidad. Los montículos estudiados se encuentran entre 18 y $25 \mathrm{~m}$ de profundidad y tienen una altura de unos 3-4 m sobre el fondo. La visibilidad durante el muestreo fue baja (7-10 m) debido a una alta concentración de partículas en suspensión.
En general, los arrecifes son moderadamente diversos (a nivel de especies y géneros). Están caracterizados por una alta cobertura de algas y una alta abundancia de corales escleractínidos y esponjas (Cuadro 2). Los géneros Montastraea, Diploria, Undaria, Agaricia, Colpophyllia, Porites, Meandrina y Stephanocoenia son los componentes dominantes de la comunidad coralina (Apéndice 1). Pseudopterogorgia y Erythropodium son los octocorales más comunes junto con esponjas tubulares, erectas, masivas e incrustantes de los géneros Aplysina, Amphimedon, Agelas, Ectyoplasia, Cliona, Spheciospongia y Niphates (Apéndices 2 y 3 ).

Playa El Coco (Isla Beata). El área muestreada está caracterizada por baja visibilidad, alta sedimentación y escalones de bajo relieve entre $10-15 \mathrm{~m}$ de profundidad a unos $200 \mathrm{~m}$ al oeste de la costa de la isla. La comunidad coralina es dispersa con una baja abundancia y cobertura de corales y una riqueza de especies intermedia (Cuadro 2). No hay formación arrecifal y los géneros Porites, Helioseris, Diploria, Siderastrea y Montastraea son los más abundantes. Octocorales y esponjas son abundantes, con una diversidad mayor pero igualmente dispersos sobre el fondo. La cobertura de algas es alta y domina la comunidad bentónica de sustrato duro.

Punta Lanza. Es una pared sumergida vertical y expuesta del arrecife fósil emergido al oeste de Isla Beata. La pared baja hasta unos $8 \mathrm{~m}$ de profundidad terminando en un fondo de arena. La localidad está caracterizada por una alta heterogeneidad espacial proporcionada por rocas calcáreas grandes que descansan sobre el fondo arenoso y que se han desprendido del arrecife fósil emergido. El área es de alta energía y generalmente turbia. La comunidad bentónica sobre la pared y a los costados y huecos de las rocas está dominada por algas verdes filamentosas e incrustantes rojas, hidrocorales y zoántidos. La riqueza y abundancia de corales, octocorales y esponjas fue la más 
baja registrada a nivel genérico y de especies (Cuadro 2). Los corales crecen de un modo incrustante con bajo relieve y algunas colonias de $M$. faveolata, $M$. cavernosa, $S$. siderea y del hidrocoral Millepora tienen una alta cobertura. Colonias pequeñas de Favia fragum son muy abundantes sobre las paredes. Colonias de tamaño intermedio $(0.5-1 \mathrm{~m})$ de $M$. faveolata se observan creciendo sobre el fondo arenoso y sobre rocas dispersas. Áreas con las mismas características geomorfológicas a lo largo de la costa oeste de Isla Beata presentan comunidades similares.

Los Carraplanes. Un arrecife de franja somero $(0-10 \mathrm{~m})$ de poca extensión, sobre un terraplén sedimentario de alta energía alrededor de unos parches arrecifales fósiles emergidos. La localidad está expuesta al oleaje y corrientes y está localizada al este de Isla Beata, en el canal entre esta la isla y la costa. La comunidad arrecifal presenta dos componentes estructurales, el arrecife principal de $A$. palmata que se desarrolla hacia el NW formando un cuña que desciende con una pendiente suave terminando en áreas arenosas y comunidades de T. testudinum, a $10 \mathrm{~m}$ de profundidad, y una formación densa de $M$. annularis al oeste formando un pequeño parche arrecifal somero (0-2 m) con altas densidades de Agaricia, Porites y coberturas altas de los zoántidos coloniales Palythoa caribbaeoroum y Zoanthus sociatus. Los corales A. palmata, M. faveolata, M. annularis, P. porites, $P$. astreoides y el hidrocoral $M$. complanata son los principales cnidarios estructurales del arrecife principal. Algas verdes y rojas filamentosas y algas calcáreas incrustantes dominan la cobertura del sustrato. Manicina areolata es muy abundante en las praderas de fanerógamas alrededor del arrecife.

La plataforma somera se extiende hasta Isla Beata y un poco al SW de los Carraplanes hay un arrecife de franja somero, expuesto al oleaje y con una plataforma somera $(0-1.5 \mathrm{~m})$ muy extensa. El frente arrecifal es una barrera formada por colonias muertas y vivas de $M$. faveolata, M. annularis, D. strigosa, S. sidera, A. palmata, P. astreoides y M.complanata, principalmente. La plataforma es espacialmente heterogénea con canales, parches y extensas áreas arenosas. Colonias de las mismas especies mencionadas arriba junto con Diploria labyrinthiformis, Agaricia agaricites, Millepora alcicornis y $P$. porites forman la estructura del arrecife trasero y los parches en la plataforma.

Cabo Falso. En esta área, una plataforma somera entre 0 y $10 \mathrm{~m}$ se extiende hasta unos $30-40 \mathrm{~m}$ de la pared vertical del arrecife emergido y luego cae abruptamente a $18 \mathrm{~m}$. A esta profundidad la plataforma se extiende hacia el oeste hasta el borde donde cae nuevamente a profundidades mayores con una pendiente pronunciada. El área está expuesta a corrientes continuas e intensas y fuerte oleaje. La plataforma somera presenta altas coberturas de algas, hidrocorales (Millepora spp.), zoántidos coloniales y la esponja endolítica Cliona tenuis (Zea and Weil 2003). Colonias dispersas de $A$. palmata, M. faveolata, D. clivosa, D. strigosa y $P$. astreoides y una alta abundancia de octocorales caracteriza esta localidad. La plataforma profunda presenta una alta heterogeneidad espacial producida por un gran número de rocas calizas de gran tamaño con grietas y huecos que descansan sobre el fondo cercano a la pendiente. Algunas rocas tienen varios metros de altura. Esta heterogeneidad espacial junto a la alta energía del área, permiten el desarrollo de una comunidad de corales, octocorales y esponjas muy diversa (Cuadro 2; Apéndices 1-3) a pesar de que las coberturas son moderadas o bajas. Las zonas crípticas y sombreadas están dominadas por esponjas de varios grupos incluyendo esponjas calcáreas. Los corales M. faveolata, M. cavernosa, D. strigosa, C. natans, A. lamarcki, U. humilis, S. siderea, P. astreoides, M. meandrites, S. intersepta son las especies más comunes. Gorgonia ventalina, Plexaura homomalla, P. flexuosa y $P$. americana son los octocorales dominantes. Hacia el borde de la plataforma insular los octocorales y esponjas en forma de vaso y colonias masivas de Montastraea incrementan en abundancias. Esta localidad presentó la 
mayor riqueza de esponjas con 68 especies de las cuales, Amphimedon, Anthosigmella, Aplysina, Agelas, Cliona, Ircina, Iotochrota, Pseudoceratina, Petrosia y Niphates son los géneros dominantes en el área. La riqueza de corales escleractínidos y octocorales es también de las más elevadas para el área.

Lanza Zo. Un complejo de bancos y montículos arrecifales alargados y bien desarrollados sobre la plataforma a unos $15 \mathrm{~m}$ de profundidad que luego forman un arrecife de franja en el talud que se extiende hasta unos $25 \mathrm{~m}$ de profundidad. El arrecife es bastante ancho (100-200 m), con poco desarrollo vertical y se extiende paralelo a la costa. Algas calcáreas como Halimeda y otras algas como Lobophora y Dictyota son muy abundantes. La comunidad coralina está dominada por especies masivas, foliosas y en forma de plato. Montastraea, Undaria, Agaricia, Porites, Madracis, Meandrina, Colpophyllia, Diploria, Stephanochoenia, Siderastrea y Helioseris son los géneros de corales escleractínidos más abundantes (Apéndice 1). La cobertura, abundancia y diversidad de éstos es mayor en el lado oeste del banco arrecifal. En este arrecife se observa por primera vez la especie de coral Mycetophyllia ressi, una especie generalmente profunda y rara en la mayoría de los arrecifes del Caribe. La abundancia y diversidad de octocorales es intermedia siendo Plexaura, Erythropodium, Pseudoterogorgia, Briaerum y Eunicea, los géneros más comunes (Apéndice 2). La riqueza y abundancia de esponjas es alta pero menor que en Cabo Falso. Esponjas tubulares, masivas y alargadas pertenecientes a los géneros Aplysina, Amphimedon, Ectyoplasia, Iotrochota, Ircina, Niphates y Pseudoceratina son bastante las más comunes (Apéndice 3).

Bahía de Águilas. Un arrecife de franja profundo (15-45 m) muy bien desarrollado que se extiende más de $300 \mathrm{~m}$ en el borde de la plataforma al oeste de Bahía de Aguilas en dirección S-N. El arrecife es ancho $(100 \mathrm{~m})$ con montículos y bandas elevadas sobre valles de arena. La parte frontal hacia el oeste está bien desarrollada con amplias áreas de colonias masivas, erectas y en forma de platos que se extienden hasta unos $45 \mathrm{~m}$ de profundidad donde comienza un fondo arenoso. Algas calcáreas (Halimeda), macroalgas frondosas $\mathrm{y}$ filamentosas (Dictyota, Lobophora) y algas calcáreas incrustantes (Peysonella) son abundantes y con altas coberturas. La diversidad, abundancia y cobertura de corales es alta, especialmente hacia el $\mathrm{N}$ y en las pendientes del oeste del arrecife. Montastraea, Undaria, Agaricia, Porites, Madracis, Meandrina, Colpophyllia, Diploria, Stephanochoenia, Siderastrea, Eusmilia, Madracis y Helioseris son los géneros dominantes (Apéndice 1). La especie rara $M$. ressi presentó una abundancia alta de colonias grandes y pequeñas en las áreas profundas del arrecife. Solenastrea bournoni fue observada por primera vez en este arrecife. M. franksi y A. lamarcki son las especies dominantes en la zona profunda del arrecife. Especies de octocorales características de áreas someras de alta energía como Gorgonia ventalina y G. flabellum son comunes en el tope del arrecife. Plexaura, Erythropodium, Briareum, Pseudoterogorgia y Eunicea fueron los géneros de octocorales dominantes (Apéndice 2). Callyspongia, Aplysina, Amphimedon, Ectyoplasia, Iotrochota, Ircina, Niphates, Cliona, y Pseudoceratina dominan la comunidad de esponjas y son muy abundantes a lo largo del arrecife (Apéndice 3).

Bahía Honda, Cabo Rojo. Un arrecife de franja profundo muy bien desarrollado con estructuras de alto relieve, valles y cañones o canales de arena y una alta riqueza y cobertura de corales. El arrecife bordea un canal de acceso de embarcaciones de gran calado a la planta de bauxita en dirección E-W y se extiende desde unos $10 \mathrm{~m}$ hasta unos $45 \mathrm{~m}$ de profundidad donde termina en un fondo arenoso de pendiente suave. La plataforma somera está cubierta por praderas de fanerógamas y parches dispersos de corales y octocorales. Algas calcáreas, macroalgas y algas calcáreas incrustantes son abundantes a lo largo de todo el arrecife. A pesar de la cercanía a la planta y 
el muelle de carga, el arrecife se encuentra en buen estado, con una cobertura alta de coral, sobre todo de colonias planas y masivas de Montastraea, Agaricia, Undaria, Colpophyllia, Diploria, Siderastrea y Porites y muy pocas colonias con daños recientes o superficies muertas. Este arrecife presentó la mayor diversidad específica de corales escleractínidos en el área, con 52 especies. Se observo una alta abundancias de colonias de la cianobacteria verde-azul Schyzotrix entre 13 y $18 \mathrm{~m}$ de profundidad. Acropora cervicornis y $P$. porites son comunes en la parte superior del arrecife y Madracis mirabilis mostró altas abundancias cubriendo áreas amplias con colonias grandes a lo largo del arrecife (Apéndice 1). Al igual que en Bahía de Águilas al sur, la especie M. ressi mostró altas abundancias de colonias grandes. S. bournoni fue observada nuevamente en este arrecife. M. franksi y $A$. lamarcki son las especies dominantes en la zona profunda del arrecife. La riqueza de octocorales y su abundancia es bastante baja en este arrecife. La riqueza de esponjas es moderadamente alta pero su cobertura y abundancias son intermedias o bajas. Aplysina, Amphimedon, Iotrochota, Ircina, Neofibularia y Pseudoceratina son los géneros comunes.

Los Frailes. Los Frailes es una pequeña isla rocosa situada a unos $20 \mathrm{~km}$ al oeste de la costa, rodeada de aguas profundas y sujeta a corrientes y oleaje fuerte. Las comunidades bentónicas se encuentran rodeadas de aguas generalmente claras, con poco sedimento. No existe una estructura arrecifal desarrollada y el sustrato es de roca caliza consolidada y muy heterogéneo espacialmente. Las comunidades están dominadas por algas, zoántidos, octocorales incrustantes y ramosos y colonias masivas y planas de corales, esponjas e hidrocorales. La alta heterogeneidad espacial es producida por una gran cantidad de rocas de gran tamaño que descansan sobre una plataforma rocosa angosta y que forman grietas, pasajes, cuevas y huecos. Se encuentran paredes verticales de hasta $10 \mathrm{~m}$ de altura sobre el fondo cubiertas por colonias del coral sin zooxantelas Tubastrea aurea en la zona N. La zona frontal hacia el E-S-E presenta colonias masivas grandes de Montastraea, Diploria y Colpophyllia, una alta abundancia de $P$. astreoides y algunas colonias de A. palmata, $P$. porites y los hidrocorales $M$. complanata y $M$. squarrosa. La riqueza de corales es alta (Cuadro 2), los géneros más abundantes son Montastraea, Undaria, Agaricia, Porites, Madracis, Meandrina, Colpophyllia, Diploria, Eusmilia, Stephanochoenia, Dichocoenia, Siderastrea, Tubastrea y Helioseris (Apéndice 1). Los octocorales son abundantes y muy diversos, los géneros más comunes son Gorgonia, Plexaura, Pseudoplexaura, Erythropodium, Briaerum y Pseudoterogorgia (Apéndice 2). La comunidad de esponjas es una de las más diversas del área con 62 especies. Los géneros más abundantes son Aplysina, Amphimedon, Iotrochota, Ircina, Cliona, Spheciospongia, Verongula y Nyphates (Apéndice 3).

\section{DISCUSIÓN}

En general, existen pocos estudios sobre la composición y abundancia de especies de corales escleractínidos, otros cnidarios y esponjas en las comunidades arrecifales de la República Dominicana. Colecciones aisladas de especímenes descansan desde mediados del siglo pasado en el Museo Nacional de Historia Natural de los Estados Unidos (Ottenwalder et al. 1999). La primera lista comprensiva de especies de coral fue el inventario de Bonnelly de Calventi (1974), la cual fue completada posteriormente con inventarios y descripciones ecológicas de localidades arrecifales principalmente en el SE, E y N del país (Geraldes y Bonnelly de Calventi 1978, Williams et al. 1983, CIBIMA 1992, Geraldes 1994, Sang 1994, 1996, Sullivan et al. 1995, Geraldes et al. 1997, 1998). Un informe preliminar (Weil 1997) y este trabajo representan el primer esfuerzo para producir un inventario detallado de especies de corales, octocorales y esponjas así como sus abundancias relativas en las áreas arrecifales del occidente del país, principalmente el Parque Nacional Jaragua. 
La alta variabilidad entre los diferentes inventarios hechos hasta la fecha puede ser una consecuencia de: (1) diferencias geomorfológicas, ecológicas, biológicas y los aspectos históricos y evolutivos que determinan las composiciones taxonómicas y la estructura de las distintas comunidades (localidades) arrecifales inventariadas; (2) las diferencias entre los diseños muestreales, máximas profundidades visitadas, y el conocimiento y experiencia sobre la taxonomía de corales y otros invertebrados marinos de los distintos autores; (3) una combinación de estos.

La problemática taxonómica de corales escleractínidos ha sido discutida en varias oportunidades (Weil 1992a, b, Fenner 1993, Weil y Knowlton 1994, Chiapone et al. 1996). La alta plasticidad morfológica de estos organismos y la falta de concenso en la clasificación de algunos grupos, hace difícil la congruencia en las identificaciones de muchas especies en distintas localidades. Por ejemplo, Geraldes y Bonnelly de Calventi (1978) y (1983) registran P. branneri en varias localidades del sur de Santo Domingo. Esta especie fue descrita para el Brasil y su presencia en el Caribe ha sido puesta en duda (Weil 1992 a, b). Diferencias morfométricas entre las dos especies, el modo reproductivo (planuladoras) y las barreras geográficas impuestas por las desembocaduras del Orinoco y el Amazonas (limitando posiblemente la dispersión de larvas y por lo tanto, el flujo genético), sugieren que la especie del Caribe es potencialmente distinta a la del Brasil (Weil 1992a, b). P. "branneri" no fue observada en ninguna de las localidades muestreadas en este estudio aún cuando el autor las buscó en los habitáculos donde se encuentra normalmente en el sur del Caribe. Colonias juveniles de $P$. astreoides y $P$. porites (fase incrustante) con coloraciones claras o azulosas son fáciles de confundir con P. "branneri" (a menos que se analice la micro-morfología del coralito). El registro de Humann (1993) para Florida es un error y la foto que presenta en su libro corresponden a un juvenil de $P$. porites y no a P. "branneri" (esto ha sido corregido en la última edición, 2002). La coloración azul es debida a pigmentos que reflejan la luz ultravioleta. Muestreos en un gran número de arrecifes en el Caribe por el autor en los últimos 20 años indican que $P$. "branneri" tiene una distribución restringida al sur y posiblemente noroeste del Caribe. Esta especie es abundante en las islas al norte de la costa de Venezuela (los Roques, La Orchila, Islas las Aves, La Blanquilla) en Bonaire y Curacao, y está registrada para Jamaica y las islas Cayman (Wells y Lang 1973, Hung 1995, Weil 1995, 2003). Registros en arrecifes de Panamá y otras localidades como Florida y Bermuda deben ser revisados. Por otro lado, P. colonensis ha sido registrada para la República Dominicana en tres publicaciones (MAMMA 1994 y Geraldes et al. 1997, 1998). Esta especie fue descubierta por el autor en la costa Caribeña de Panamá en 1986 y fue descrita posteriormente por Zlatarski (1990). La especie puede confundirse fácilmente con formas crípticas y planas de $P$. astreoides. Observaciones en muchas localidades del Caribe en los últimos 10 años sugieren que la especie es posiblemente endémica para las costas caribeñas de Panamá y Costa Rica (Weil 1992a, b). Muestras identificadas como P. colonensis que fueron colectadas en Belize y México y enviadas al autor para verificación, resultaron ser $P$. astreoides. Los nombres Tubastrea aurea y Tubastrea coccinea son generalmente utilizados como sinonimias. El último nombre es el más antiguo y ha sido utilizado ampliamente en inventarios de especies de corales en el Caribe (Cairns 1982, 2000, Sullivan et al. 1995, Geraldes et al. 1997, 1998, Fenner 2001). Diferencias genéticas (isoenzimas) y morfológicas (morfometría) entre poblaciones de Tubastrea en Venezuela y la costa Pacífica de Panamá, indican que estas son dos especies distintas (Weil y Brunneti, datos no publicados). Estos problemas tienden a aumentar los números totales de especies registradas para ciertas regiones y confunde los patrones de distribución geográfica (Budd et al. 1994). Los registros de especies con taxonomía dudosa como Solenastrea hyades (CMC 1994, Sang 1996), P. colonensis y P. "branneri" (Geraldes y Bonnelly de Calventi 1978, Williams 1983, 
CMC 1994, MAMMA 1994, Geraldes et al. 1997, Geraldes 2003) para la República Dominicana deben ser revisados.

Los resultados de este estudio representan las listas más completas de especies de corales, octocoral y esponjas para la República Dominicana. Los resultados indican que ocho especies de corales, 21 especies de octocorales y 59 especies de esponjas constituyen nuevos registros para la República Dominicana [si consideramos las listas más completas de Bardales (Williams et al. 1983)]. Además, los resultados indican que los arrecifes del Parque Nacional Jaragua y del área circundante tienen la mayor riqueza de corales de la República Dominicana (cuando se comparan con otras localidades citadas en la literatura), con un total de 56 especies de corales escleractínidos (51 con zooxantelas y cinco sin zooxantelas). Esta es, sin duda, una lista todavía incompleta ya que hace falta revisar más detalladamente la composición de especies crípticas (sin zooxantelas) en las comunidades no muestreadas, zonas más profundas y zonas con condiciones extremas. Dos especies conspicuas y abundantes en otras áreas arrecifales de la República Dominicana y el norte del Caribe pero que no se observaron en las localidades muestreadas en este estudio son Dendrogyra cylindrus y Porites divaricata. Esto no significa que estas especies no se encuentren en el área ya que las limitaciones de tiempo no permitieron un muestreo más extensivo. Por otro lado, Mycetophyllia ressi, una especie generalmente rara o ausente en la mayoría de los arrecifes caribeños y Madracis senaria (una especie generalmente confundida con $M$. pharensis y M. decactis), son muy comunes en al menos cuatro de las nueve localidades muestreadas, presentando una alta abundancia de colonias bien desarrolladas. Especies poco comunes con una distribución restringida por hábitat o gradiente de profundidad como Oculina sp., Cladocora arbuscula y $P$. divaricata no se observaron en este estudio pero pueden estar presentes en el área.

A nivel geográfico, la diversidad coralina es de las más altas registradas para el norte del
Caribe pero todavía está por debajo de áreas como Venezuela, Panamá, Bonaire, Belize, islas Cayman y Jamaica (Wells y Lang 1973, Weil 1985, Holst y Guzmán 1993, Guzmán 2003, Weil 2003, Weil obs. pers.). La diversidad coralina de la República Dominicana es comparable con la de Cuba (Zlatarski y Estalella 1982), Puerto Rico (Almy y CarrionTorres 1963, Coenaga y Cintrón 1979, Weil, obs. pers.), Bahamas (Weil, obs. pers.), Florida (Weil et al. obs. pers.), Belize (Cairns 1982), las Islas Vírgenes y el Golfo de México (Chiaponne et al. 1996). La composición de las comunidades profundas y someras es un poco distinta a las áreas arrecifales del sur y el oeste del Caribe donde Agaricia lamarcki, A. grahamae, A. undata, U. humilis y $U$. purpurea y varias especies de Madracis dominan estos hábitats junto con $M$. franksi, mientras que Undaria tenuifolia, Acropora cervicornis, $P$. furcata y $P$. porites dominan los hábitats someros junto a $M$. faveolata y M. annularis. Los géneros Montastraea, Colpophyllia, Diploria, Porites y Siderastrea son los principales constructores de áreas arrecifales a profundidades intermedias al igual que en la mayoría de los arrecifes del Caribe.

En general, el Parque Nacional Jaragua en el oeste de la República Dominicana presenta arrecifes bien deasarrollados con las diversidades de corales, octocorales y esponjas más altas registradas para la isla. La diversidad y abundancias de esponjas es particularmente elevada y representa un grupo interesante para futuras investigaciones. Una recomendación de este estudio es que la zona debe ser protegida contra desarrollos turísticos, minería y otras actividades costeras con potencial para alterar las condiciones ambientales del área. Las actividades pesqueras deben ser reguladas ya que nuestras observaciones indican una abundancia y riqueza de peces muy baja para comunidades arrecifales como las observadas en este estudio. Durante nuestra estadia en el área pudimos observar lo intensivo y extensivo de las actividades pesqueras utilizando redes de ahorque de más de $500 \mathrm{~m}$ de largo constantemente 
extendidas alrededor o sobre los arrecifes, pesca con arpones utilizando compresores de aire para pintar, etc. Esta actividad de pesca no controlada seguramente ha mermado las poblaciones de peces herbívoros (muy pocos peces loro o peces cirujanos observados) lo cual puede ser la causa de los problemas de algas sobrecreciendo a muchas colonias de coral. El área carece de ríos por lo que enriquecimiento de nutrientes no parece justificar el crecimiento de algas. La falta de especies carnívoras (meros, pargos, barracudas, etc,) podría ser la causa de las altas abundancias observadas de especies como las damiselas. Esto trae como consecuencia un incremento en la competencia por territorios y las damiselas han comenzado a dañar áreas de coral vivo (M. faveolata) para sus campos de algas.

\section{AGRADECIMIENTOS}

Al Grupo Jaragua y Barbara Reveles González por darme la oportunidad de participar en la Segunda Expedición exploratoria al Parque Nacional Jaragua y financiar este estudio. A Yolanda León, Claudio y Yira Rodríguez por su ayuda y excelente disposición durante el trabajo de campo. A la tripulación del B/I Mago del Mar por su ayuda logística y colaboración durante la expedición. Al Departamento de Ciencias Marinas de la Universidad de Puerto Rico y a los revisores por sus comentarios y mejoras del manuscrito original.

\section{RESUMEN}

El Parque Nacional Jaragua está localizado en un área remota al SW de la República Dominicana. La actividad humana se limita a pesquerías y minería. Las principales formaciones arrecifales incluyen (a) arrecifes de banco (montículo) creciendo sobre la plataforma en bandas paralelas, separadas por canales de arena y en dirección $\mathrm{SE}-\mathrm{NW}$, entre 12 y $25 \mathrm{~m}$ de profundidad, (b) arrecifes de franja profundos que se desarrollan en los bordes de la plataforma entre 10 y $45 \mathrm{~m}$ de profundidad, y (c) parches arrecifales y comunidades coralinas con poca o ninguna estructura arrecifal en áreas cercanas a la costa y plataformas someras. Nueve localidades fueron muestreadas intensivamente entre Cabo Beata y Bahía Honda mediante buceo autónomo. Se nadó en un patrón de zig-zag (50 $\mathrm{m}$ a cada lado) desde el fondo $(30 \mathrm{~m})$ hasta la zona somera en los arrecifes frangeantes. Se uso el mismo patrón pero a lo largo (por $500 \mathrm{~m}$ ) cubriendo el ancho de las bandas en los arrecifes de montículo. Las abundancias relativas y la diversidad de corales escleractínidos, octocorales y esponjas se estimaron en estas bandas. Las esponjas presentaron la mayor diversidad con 83 especies en 50 géneros, seguidas por los corales escleractínidos con 56 especies en 26 géneros y los octocorales con 47 especies en 15 géneros. Nuevos registros para el área incluyen ocho especies de corales, 21 de octocorales y 59 de esponjas. La diversidad, abundancia y distribución de especies de cada grupo varió entre localidades. En general, las localidades del norte y la isla de Los Frailes mar afuera, mostraron las mayores diversidades y abundancias de los tres grupos. En general, el Parque Nacional Jaragua presenta las diversidades más altas de corales, octocorales y esponjas registradas para la República Dominicana y están entre las más diversas para el norte del Caribe. Se recomienda una protección integral del área que incluya una regulación substancial (o prohibición) de las actividades pesqueras.

Palabras clave: diversidad, abundancia relativa, arrecifes, octocorales, esponjas, Parque Nacional Jaragua, República Dominicana, Mar Caribe.

\section{REFERENCIAS}

Almy, C.C. \& C. Carrion-Torres. 1963. Shallow-water stony corals of Puerto Rico. Caribb. J. Sci. 3: 133162 .

Bayer, F.M. 1973. The shallow-water Octocorallia of the West Indian region. Stu. Fauna Curacao 12: 1-373.

Bonnelly de Calventi, I. 1974. Corales pétreos de la República Dominicana. Estudios de biología pesquera dominicana. Universidad Autónoma de Santo Domingo, República Dominicana. p. 65-69.

Budd, A.F., T. Stemann \& K. Johnson. 1994. Stratigraphic distributions of genera and species of Neogene and Recent Caribbean reef corals. J. Paleont. 69: 951977.

Cairns, S.D. 2000. Stud. Nat. Hist. Carib. Reg. 75: 1-235.

Cairns, S.D. 1982. Stony Corals of Carrie Bow Cay, Belize. In K. Ruetzler \& I. Macintyre (eds.). The Atlantic Barrier Reef Ecosystem of Carrie Bow Cay, Belize. Smith. Contr. Mar. Sci. 12: 532 p.

Chiaponne, M.K. M. Sullivan \& C. Lott. 1996. Hermatypic scleractinian corals of the southwestern Bahamas, a comparison to western Atlantic reef systems. Carib. J. Sci. 32: 1-13. 
CIBIMA. 1992. Los Cnidarios o Celenterados. Estudio Preliminar sobre Biodiversidad Costera y Marina de la República Dominicana. Alfa-Omega, Sto. Domingo, República Dominicana. pp. 277-288.

CMC. 1994. Rapid ecological assessment. Parque Nacional del Este, Dominican Republic. Technical Report of the Caribbean Conservation Science Center, University of Miami, Florida, EEUU. 365 pp.

Fenner, D. 1993. Species distinction among several species of Caribbean stony corals. Bull. Mar. Sci. 53: 1099-1116.

Fenner, D. 2001. Bull. Mar. Sci. 69: 1175-1189.

Geraldes, F.X. 1994. Iniciativa para la conservación de los arrecifes del Caribe, República Dominicana. Centro de Investigaciones de Biología Marina. Universidad Autónoma de Santo Domingo, República Dominicana. 75 p.

Geraldes, F.X. \& I. Bonnelly de Calventi. 1978. Los arrecifes de coral de la costa sur de la República Dominicana. Ecología y Conservación. En Conservación y Ecodesarrollo. Centro de Investigaciones de Biología Marina. Universidad Autónoma de Santo Domingo, República Dominicana. p. 108-115.

Geraldes, F.X., C. Mateo, G. Rosado, V. Alvares, E.J. Marcano, M. Vega, S. Navarro, E. Pugibet, M.P. Pérez, H. Ramírez, V. Rivas, Y. Rodríguez, D. Montero, M. Asunción \& C. Montero. 1998. La diversidad biológica de los ecosistemas marinos del Parque Nacional Montecristi. Reporte final al Proyecto GEF-PNUD/ ONOPLAN, CIBIMA, Universidad Autónoma de Santo Domingo, República Dominicana. 36 p.

Geraldes, F.X., M. Vega, E. Pugibet, R.E. Torres, Y. Rodríguez, L. Almánzar \& D. Guerrero. 1997. Estudio y prospección de las condiciones ecológicas y uso del Parque Nacional Submarina La Caleta. Fundación Dominicana Pro-investigación y Conservación de los Recursos Marinos. MAMMA, República Dominicana. 61 p.

Geraldes, F.X. 2003. The coral reefs of the Dominican Republic; p. 303-330. In J. Cortés (ed.). Latin American Coral Reefs, Elsevier, Amsterdam, Holanda.

Goenaga, C. \& C. Cintrón. 1979. Inventory of the Puerto Rican coral reefs. Coastal Zone Management Report. DNR- Puerto Rico. San Juan, Puerto Rico. 200 p.

Guzmán, H. 2003. Caribbean coral reefs of Panama: Present status and future perspectives; p. 241-274. In J. Cortés (ed.). Larin American Coral Reefs. Elsevier, Amsterdam, Holanda.

Holst, I. \& H.M. Guzmán 1993. Lista de corales hermatípicos (Anthozoa, Scleractinia, Hydrozoa, Milleporina) a ambos lados del Istmos de Panamá. Rev. Bio. Trop. 41: 871-875.

Humann, P. 1993. Reef Coral Identification. New World Publications, EEUU. 239 p.

Hung, M.F. 1985. Los corales pétreos del Parque Nacional Archipiélago de Los Roques. Tesis de Licenciatura. Facultad de Ciencias, Escuela de Biología, Universidad Central de Venezuela, Caracas, Venezuela. 204 p.

MAMMA. 1994. Estudio y prospección de las condiciones ecológicas y uso del Parque Nacional Submarina La Caleta. R. Dominicana. Informe final Programa de Apoyo de Areas Protegidas. Fund. Dominicana Pro-investigación y Conservación de los Recursos Marinos. MAMMA, República Dominicana. 61 p.

Ottenwalder, J., A.A. Herrera \& L. Betancourt. 1999. Corales Pétreos; 200 p. In Evaluación de la biodiversidad marina de la República Dominicana y Haití (Hispaniola) con comentarios sobre distribución, hábitats y situación actual. Santo Domingo, República Dominicana.

Sang, L. 1994. Arrecifes de coral; p. 73-93. In Caracterización de Ecosistemas Costeros y Marinos en la Bahía de Samaná. Centro para la Investigación y Ecodesarrollo de la Bahía de Samaná y su Entorno. Santo Domingo, República Dominicana.

Sang, L. 1996. Estudio de los arrecifes de coral de la costa norte de la Península de Samaná. Centro para la Investigación y Ecodesarrollo de la Bahía de Samaná y su Entorno. Santo Domingo, República Dominicana. 97 p.

Sullivan, K.M., M. Chiappone, R. Sluka, E.F. Schmitt \& G.A. Delgado. 1995. Manual of assessment and monitoring methods. Scientific investigations in East National Park, Dominican Republic, The Nature Conservancy. Santo Domingo, República Dominicana. $170 \mathrm{p}$.

Stemann, T.A. 1991. Evolution of the reef coral family Agariciidae (Anthozoa: Scleractinia) in the Neogene through Recent of the Caribbean. Ph.D dissertation. Univ. of Iowa, Iowa, EEUU. 321 p.

Weidenmeyer, F. 1978. Shallow-water sponges of the Western Bahamas. Birkhauser, Alemania. 287 p.

Weil, E. 1985. Corales escleractínidos del Parque Nacional Archipiélago de Los Roques. Informe Técnico Fundación Científica Los Roques, Caracas, Venezuela. 5 p.

Weil, E. 1992a. Genetic and morphological variation in Porites (Anthozoa, Scleractinia) across the Isthmus of Panama. Ph.D dissertation, University of Texas at Austin, Austin, Texas, EEUU. 238 p. 
Weil, E. 1992b. Genetic and morphological variation in Caribbean and eastern Pacific Porites (Anthozoa, Scleractinia). Preliminary results. Proceed. 7th Int. Coral Reef Symp. Guam.Vol. 2: 643-656.

Weil, E. 1997. Coral, octocoral and sponge diversity in the reefs of the Jaragua National Park. Report to the Jaragua Group. Project on the Biodiversity of the Coastal and Marine Environments of the Dominican Republic. Santo Domingo, República Dominicana. $11 \mathrm{p}$.

Weil, E. 2003. Coral and coral reefs of Venezuela; p. 303330. In J. Cortés (ed.). Latin American Coral Reefs, Elsevier, Amsterdam, Holanda.

Weil, E. \& N. Knowlton. 1994. A Multi-Character analysis of the Caribbean coral Montastraea annularis (Ellis \& Solander 1786) and its two sibling species $M$. faveolata (Ellis \& Solander 1786) and M. franksi (Gregory 1895). Bull. Mar. Sci. 55: 151-175.

Wells, J.W. 1973. New and old scleractinian corals from Jamaica. Bull. Mar. Sci. 23: 16-55.
Wells, J.W. \& J.C. Lang. 1973. Systematic list of Jamaican shallow-water Scleractinia. Bull. Mar. Sci. 23: 55-58.

Williams, E. H., I. Clavijo, J.J. Kimmel, P.L. Colin, C. Díaz, A.T. Bardales, R.A. Armstrong, L. B. Williams, R.H. Boulon \& J.R. García. 1983. A checklist of marine plants and animals of the south coast of the Dominican Republic. Carib. J. Sci. 19: 39-54.

Zea, S. 1987. Esponjas del Caribe Colombiano. Catálogo Científico. Santo Domingo, República Dominicana. 286 p.

Zea, S. \& E. Weil. 2003. Taxonomy of the Caribbean excavating sponge species complex Cliona caribbaea - C. aprica - C. langae (Porifera, Hadromerida, Clionaidae). Carib. J. Sci. 39: 348-370.

Zlatarski, V. 1990. Porites colonensis, new species of stony coral (Anthozoa: Scleractinia) off the Caribbean coast of Panama. Proc. Biol. Soc. Wash. 103: 257-264.

Zlatarski, V. \& E.M. Estalella. 1982. Les Scleractiniaries de Cuba avec des données sue les organismes associés, editions de L'Academie bulgare des sciences, Sofia, Bulgaria. Chapter 1: $472 \mathrm{p}$. 


\section{APÉNDICE 1}

Especies y abundancias relativas de corales escleractínidos e hidrocorales en las distintas localidades muestreadas del Parque Nacional Jaragua en la costa SO de la República Dominicana

\section{Especies}

Acropora palmata

A. cervicornis

A. prolifera

Undaria agaricites

U.humilis

U purpurea

$U$ danae

U carinata

Agaricia fragilis

A. lamarcki

A. grahamae

Agaricia sp.

Helioseris cucullata 1

H. cucullata2@

Porites porites

P. furcata

P. astreoides B (brown)

P. astreoides Y (yellow)

Madracis decactis

M. mirabilis

M. pharensis $\mathrm{P} *$

M. pharensis L @

M. senaria

Diploria strigosa

D. clivosa

D. labyrinthyformis

Diploriasp.@

Colpophyllia natans

C.breviserialis@

C. amaranthus

Meandrina meandrites

M. memorialis

M. "brasiliensis"@
Arrecifes

GRUM PCOC CARRA PLAN CFAL LZO BAGUI BHONDA FRA

$+++\quad++$

$++++$

$\begin{array}{cc}++ & ++ \\ + & + \\ ++ & ++ \\ ++ & \\ + & \end{array}$

$\begin{array}{cc}++ & + \\ ++ & + \\ ++ & + \\ ++ & + \\ ++ & ++\end{array}$

$++$

$++$

$++$

$++$

$+$

$++$

$++$

$++$

$+++$

$+++$

$++$

$+$

$\begin{array}{ll}++ & ++\end{array}$

$+$

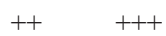

$++$

$+$

$+\quad++$

$++\quad++$

$++$

$+$

$+++\quad+++$

$+$

$++$

+++

+++

$+++$

$+++$

$+$

$+++$

$+++$

$+$

$+++$

$+++$

$+$

$+++\quad+++$

$+$

$++$

$+++$

$++$

$+++$

$+++$

$++++$

$+++$

$+++$

$+++$

$+++\quad+++$

$+++$

$+++$

$++$

$++$

$++$

$+$

$+$

$+$

$+$

++ $\quad++\quad++$

$++++$

$++$

$++\quad+$$$
++
$$

+++

$++$

$++$

$++$

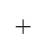

$+$

$+++$

$+++$

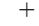

$+$

$+$

$+\quad++$

+++ +++

$++$

$+$

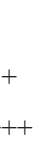

\section{(n)}




\section{APÉNDICE 1 (Continuación)}

Especies y abundancias relativas de corales escleractínidos e hidrocorales en las distintas localidades muestreadas del Parque Nacional Jaragua en la costa SO de la República Dominicana

Especies

Arrecifes

\begin{tabular}{|c|c|c|c|c|c|c|c|c|c|}
\hline & GRUM & PCOC & CARRA & PLAN & CFAL & LZO & BAGUI & BHONDA & FRA \\
\hline Montastraea annularis & + & ++ & +++ & + & +++ & +++ & +++ & +++ & +++ \\
\hline M. faveolata & +++ & ++ & ++ & ++ & +++ & ++ & ++ & + & ++ \\
\hline M. franksi & +++ & ++ & + & & +++ & +++ & +++ & +++ & ++ \\
\hline M. cavernosa 1 & +++ & +++ & ++ & ++ & +++ & +++ & +++ & +++ & ++ \\
\hline M. cavernosa $2 @$ & + & + & & & ++ & +++ & ++ & ++ & + \\
\hline Solenastrea bournoni & & + & & & & & + & + & \\
\hline Siderastrea siderea & ++ & +++ & +++ & ++ & +++ & ++ & ++ & ++ & +++ \\
\hline S. radians & & & + & ++ & ++ & & & & ++ \\
\hline Dichocoenia stockesii & + & ++ & & + & ++ & + & ++ & + & + \\
\hline D. stellaris & ++ & + & & & ++ & + & + & + & +++ \\
\hline Eusmilia fastigiata & ++ & + & & & ++ & + & ++ & ++ & +++ \\
\hline Favia fragum & & & & +++ & & & & & ++ \\
\hline Manicina aerolata & & & ++ & & & & & + & \\
\hline M. mayori & & & & & & + & + & + & \\
\hline Mycetophyllia alicia & ++ & + & & & + & + & ++ & ++ & + \\
\hline M. lamarckiana & +++ & ++ & & & ++ & +++ & ++ & +++ & ++ \\
\hline M. danaana & + & & & & + & ++ & + & ++ & ++ \\
\hline M. ferox & ++ & & & & + & + & ++ & + & + \\
\hline M. ressi & & & & & & ++ & ++ & + & \\
\hline Scolymia cubensis & + & + & & & + & ++ & + & + & + \\
\hline S. lacera & & & & & + & & + & + & \\
\hline S.wellsi@ & + & & & & + & + & + & + & + \\
\hline Isophyllastrea rigida & ++ & ++ & ++ & & +++ & +++ & & + & ++ \\
\hline Isophyllia sinuosa & & & + & & & + & & + & \\
\hline Mussa angulosa & + & + & & & + & + & + & ++ & \\
\hline $\begin{array}{l}\text { Stephanocoenia } \\
\text { intersepta }\end{array}$ & +++ & +++ & & & +++ & ++ & +++ & +++ & + \\
\hline Stephanocoenia.sp.@ & & & & & ++ & + & ++ & + & + \\
\hline Phyllangia americana* & + & + & + & + & ++ & + & + & + & + \\
\hline Astrangia solitaria* & + & + & + & + & + & + & + & + & + \\
\hline Tubastrea aurea* & + & & + & + & ++ & ++ & + & ++ & +++ \\
\hline Rhyzosmilia maculata* & + & + & & & + & & & + & + \\
\hline Millepora alcicornis & + & + & +++ & ++ & ++ & ++ & ++ & ++ & ++ \\
\hline
\end{tabular}




\section{APÉNDICE 1 (Continuación)}

Especies y abundancias relativas de corales escleractínidos e hidrocorales en las distintas localidades muestreadas del Parque Nacional Jaragua en la costa SO de la República Dominicana

\begin{tabular}{|c|c|c|c|c|c|c|c|c|c|}
\hline \multirow{2}{*}{ Especies } & \multicolumn{9}{|c|}{ Arrecifes } \\
\hline & GRUM & $\mathrm{PCOC}$ & CARRA & PLAN & CFAL & LZO & BAGUI & BHONDA & FRA \\
\hline M. complanata & & + & ++ & & ++ & & + & ++ & ++ \\
\hline M. squarrosa & & & & & ++ & & & ++ & ++ \\
\hline Stylaster roseus & & & & & & + & & & + \\
\hline Corales escleractínidos & 45 & 38 & 24 & 19 & 50 & 46 & 48 & 52 & 49 \\
\hline con zooxantelas & 40 & 35 & 21 & 16 & 45 & 42 & 44 & 47 & 44 \\
\hline$*=\sin$ zooxantelas & 5 & 3 & 3 & 3 & 5 & 4 & 4 & 5 & 5 \\
\hline Géneros de corales & 23 & 22 & 14 & 12 & 23 & 23 & 24 & 25 & 23 \\
\hline Hidrocorales & 1 & 2 & 2 & 1 & 3 & 2 & 2 & 3 & 4 \\
\hline
\end{tabular}

+++ = abundante; $++=$ común; += rara. @ = taxa con un estatus taxonómico dudoso. * = especies sin zooxanthelas.

Localidades: $\mathrm{GRU}=$ Grumelandia; $\mathrm{PCOC}=$ Playa Coco; CARRA $=$ Carraplanes; PLAN $=$ Punta Lanza; $\mathrm{CFAL}=\mathrm{Cabo}$ Falso; LZO = Lanza ZO; BAGUI = Bahía de Aguilas; BHONDA= Bahía Honda en Cabo Rojo; FRA = Los Frailes. 


\section{APÉNDICE 2}

Especies de octocorales y sus abundancias relativas observadas en las diferentes localidades del Parque Nacional Jaragua, República Dominicana

Especies

Briaerum asbestinum 1

B. asbestinum 2

Erythropodium caribaeorum

Eunicea laxispica

E. succinea $\mathrm{S}$

E. succinea $\mathrm{P}$

E. lascinata

E. asperula

E. fusca

E. mammosa

E. laxa

E. clavigera

E. tourneforti 1

E. tourneforti 2

E. calyculata

E. palmieri

E. knighty

E. sp.

Gorgonia ventalina

G. flabellum

G. mariae

Iciligorgia schrammi

Muricea muricata

M. laxa

M. elongata

Muriceopsis flavida

Plexaura homomalla

P. flexuosa

P. kuna

Plexaurella dichotoma

P. grises

P. nutans

Pseudoplexaura flagellosa
Arrecifes

GRUM PCOC CARRA PLAN CFAL LZO BAGUI BHONDA FRA

$++\quad++$

$++$

$+$

$++$

$+$

$++$

$+$

$++$

$$
+
$$$$
++
$$$$
++
$$$$
+
$$$$
+
$$$$
++
$$$$
\begin{array}{cc}
+ & ++ \\
++ & ++
\end{array}
$$$$
+++
$$$$
+++
$$$$
+
$$$$
++
$$$$
++
$$$$
+
$$$$
+
$$$$
+
$$$$
+
$$$$
++\quad++
$$
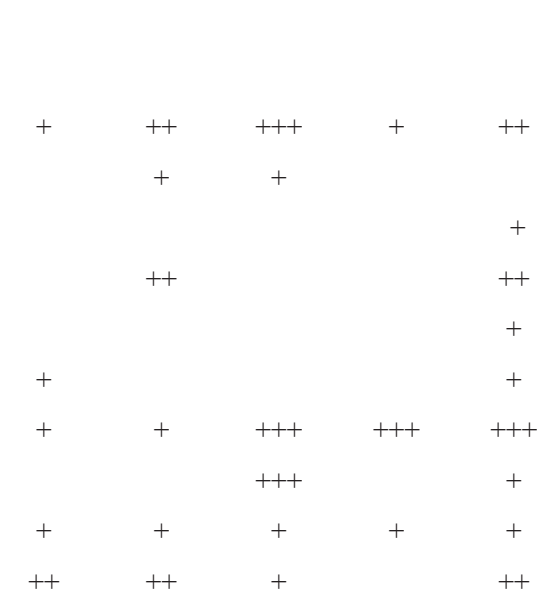

$++$

$+$

$+$
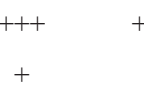

$+$

$++\quad++$

$+\quad++$$$
+
$$

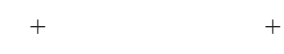

\begin{tabular}{|c|c|c|c|c|c|c|c|c|}
\hline \multirow[t]{2}{*}{+} & ++ & & & + & + & ++ & & + \\
\hline & +++ & +++ & ++ & +++ & +++ & ++ & ++ & +++ \\
\hline & ++ & ++ & ++ & +++ & + & ++ & + & +++ \\
\hline & ++ & + & & ++ & & + & & ++ \\
\hline+ & ++ & + & & ++ & + & ++ & ++ & ++ \\
\hline & ++ & & & + & + & + & & + \\
\hline & +++ & & & + & + & +++ & & + \\
\hline & + & + & & + & ++ & + & + & + \\
\hline
\end{tabular}




\section{APÉNDICE 2 (Continuación)}

Especies de octocorales y sus abundancias relativas observadas en las diferentes localidades del Parque Nacional Jaragua, República Dominicana

\begin{tabular}{|c|c|c|c|c|c|c|c|c|c|}
\hline \multirow{2}{*}{ Especies } & \multicolumn{9}{|c|}{ Arrecifes } \\
\hline & GRUM & PCOC & CARRA & PLAN & CFAL & LZO & BAGUI & BHONDA & FRA \\
\hline P. porosa & & ++ & + & & + & + & ++ & + & + \\
\hline P. crucis & & & & & + & & + & & + \\
\hline P. wagenari & & + & & & + & & ++ & ++ & + \\
\hline Pseudoterogorgia americana & +++ & +++ & +++ & + & +++ & +++ & +++ & ++ & +++ \\
\hline P. kallos & ++ & ++ & + & + & + & + & ++ & + & + \\
\hline P. acerosa & + & + & + & + & ++ & & + & & ++ \\
\hline P. bipinnata & + & ++ & & & ++ & + & ++ & & ++ \\
\hline$P$. sp. & & + & + & & ++ & & + & & ++ \\
\hline Pterogorgia citrina & & + & & & & & + & & \\
\hline P. ancepts & & ++ & & & ++ & + & + & & ++ \\
\hline P. guadalupensis & + & + & & & ++ & ++ & ++ & ++ & ++ \\
\hline Diodogorgia nudiliferos & & & & & + & + & + & + & + \\
\hline Ellisella barbadensis & + & & & & + & & + & & + \\
\hline Swiftia exserta & & & & & + & + & + & & + \\
\hline Especies de octocorales & 19 & 31 & 25 & 24 & 41 & 28 & 44 & 22 & 41 \\
\hline Géneros de octocorales & 11 & 10 & 10 & 6 & 15 & 12 & 15 & 10 & 15 \\
\hline
\end{tabular}

Localidades: $\mathrm{GRU}=$ Grumelandia; $\mathrm{PCOC}=$ Playa Coco; CARRA $=$ Carraplanes; PLAN $=$ Punta Lanza; $\mathrm{CFAL}=\mathrm{Cabo}$ Falso; LZO = Lanza ZO; BAGUI = Bahía de Aguilas; BHONDA= Bahía Honda en Cabo Rojo; FRA = Los Frailes. 


\section{APÉNDICE 3}

Lista y abundancia relativa de las especies de Porifera (esponjas) observadas en las diferentes localidades del Parque Nacional Jaragua, República Dominicana

Especies

\begin{tabular}{|c|c|c|c|c|c|c|c|c|c|}
\hline Especies & GRUM & $\mathrm{PCOC}$ & CARRA & PLAN & CFAL & $\mathrm{LZO}$ & BAGU & BHONDA & FRA \\
\hline Amphimedon compressa & +++ & +++ & ++ & & +++ & +++ & +++ & +++ & ++ \\
\hline A. erina & & & + & & & & + & + & + \\
\hline Anthosigmella varians & +++ & ++ & ++ & & +++ & +++ & ++ & + & + \\
\hline Aplysina fulva & + & + & & & ++ & ++ & ++ & ++ & ++ \\
\hline A. fistularis & +++ & & ++ & +++ & + & + & + & + & + \\
\hline A. cauliformis & +++ & ++ & + & & +++ & +++ & +++ & +++ & +++ \\
\hline A. archeri & ++ & & + & & ++ & ++ & + & + & + \\
\hline A. lacunosa & & +++ & & & +++ & +++ & ++ & + & ++ \\
\hline Agelas conifera & ++ & ++ & & & +++ & +++ & + & + & ++ \\
\hline A. weidenmyeri & ++ & & + & & ++ & + & + & & + \\
\hline A. clathrodes & + & ++ & + & & +++ & ++ & ++ & + & + \\
\hline A. schmitii & + & + & & & + & & & + & + \\
\hline A. dispar & ++ & ++ & & ++ & ++ & & + & & \\
\hline Artemisina melana & & + & & & + & & & & \\
\hline Adocia implexiformis & & & & & + & & & & \\
\hline Aplysilla glacilis & & & & & + & + & & & \\
\hline Calix podatypa & + & & & & ++ & + & ++ & + & \\
\hline Callyspongia vaginalis & ++ & ++ & + & & ++ & ++ & +++ & ++ & ++ \\
\hline C. vaginalis 2 & & + & & & + & & + & + & ++ \\
\hline C. plicifera & & & & & ++ & + & + & & + \\
\hline C. armigera & & + & & & + & & ++ & ++ & ++ \\
\hline C.palas & & + & & & + & & & & \\
\hline Clathria sp. & & & & & & + & + & + & + \\
\hline Cliona sp. & & + & + & & + & & ++ & ++ & ++ \\
\hline C. aprica & +++ & ++ & + & +++ & ++ & ++ & ++ & ++ & ++ \\
\hline C. langae & +++ & ++ & + & + & ++ & ++ & + & + & + \\
\hline C. delitrix & ++ & +++ & & & ++ & ++ & ++ & ++ & +++ \\
\hline C. lampa & & + & & & + & + & + & ++ & + \\
\hline Cribochalina vasculum & +++ & + & + & & ++ & ++ & + & & + \\
\hline Cynachira sp. & ++ & & & & + & ++ & & & \\
\hline Desmapsamma anchorata & & + & + & & + & & ++ & + & ++ \\
\hline Diplastrella sp. & + & & & & + & + & + & + & + \\
\hline D. megastellata & & & + & & + & + & + & ++ & + \\
\hline
\end{tabular}




\section{APÉNDICE 3 (Continuación)}

Lista y abundancia relativa de las especies de Porifera (esponjas) observadas en las diferentes localidades del Parque Nacional Jaragua, República Dominicana

Especies

\begin{tabular}{|c|c|c|c|c|c|c|c|c|c|}
\hline . & GRUM & $\mathrm{PCOC}$ & CARRA & PLAN & CFAL & LZO & BAGU & BHONDA & FRA \\
\hline Dysidea janiae & + & & & & ++ & & + & & \\
\hline D. etheria & + & & & & ++ & & + & & + \\
\hline Ectyoplasia ferox & +++ & ++ & + & & ++ & +++ & ++ & ++ & ++ \\
\hline Geodia neptuni & ++ & & & & ++ & + & ++ & ++ & + \\
\hline Halisarca sp. & + & & & & + & + & & & \\
\hline Holpsamma helwigi & & + & & & ++ & + & ++ & +++ & +++ \\
\hline Hyatella intestinalis & & + & & + & & & ++ & ++ & + \\
\hline Hyrtios proteus & + & + & + & & + & ++ & ++ & ++ & ++ \\
\hline Iotrochota birotulata & + & & + & & ++ & +++ & +++ & +++ & +++ \\
\hline Ircina felix & ++ & +++ & + & ++ & +++ & +++ & +++ & +++ & ++ \\
\hline I. strobilina & + & +++ & ++ & & ++ & + & + & ++ & +++ \\
\hline I. campana & & & & & ++ & & + & & \\
\hline Leucandra aspera & & & & & + & + & & & + \\
\hline Leucerta barbata & & & & & + & & & & + \\
\hline Monachora unguifera & & ++ & + & & + & + & ++ & + & + \\
\hline M. barbadensis & + & & & & + & ++ & + & + & + \\
\hline Mycale laevis & & + & + & & + & & ++ & ++ & + \\
\hline M. laxissima & & & & & + & + & & & \\
\hline Mycale styx & & & & & + & + & & & + \\
\hline M. ardnt & & & & & + & & & & + \\
\hline Neofibularia nolitangere & ++ & & + & & + & + & ++ & + & ++ \\
\hline Niphates alba & & & & & & & ++ & +++ & +++ \\
\hline N. erecta & +++ & ++ & ++ & ++ & +++ & +++ & +++ & +++ & ++ \\
\hline N. digitalis & & ++ & & & ++ & ++ & + & + & ++ \\
\hline Spongia obscura & & & & & & & + & ++ & \\
\hline Pellina carbonaria & & & & & & & + & & \\
\hline Pretosia pellasarca & & & & & + & & & + & + \\
\hline P. weinbergi & & + & + & ++ & +++ & & ++ & + & + \\
\hline Phorbas amaranthus & ++ & ++ & + & + & + & ++ & ++ & + & ++ \\
\hline Plesiastrea sp. & & & & & & & + & ++ & + \\
\hline Pseudaxinella luneacarta & & ++ & + & & ++ & & ++ & ++ & ++ \\
\hline Pseudoceratina crassa & ++ & ++ & & & +++ & +++ & ++ & +++ & ++ \\
\hline Ptilocaulis wallpersi & & & & & + & & & & \\
\hline
\end{tabular}




\section{APÉNDICE 3 (Continuación)}

Lista y abundancia relativa de las especies de Porifera (esponjas) observadas en las diferentes localidades del Parque Nacional Jaragua, República Dominicana

Especies

Plakortis halichondriales

P. angulospiculatus

Siphonodictyon

coralliphagum

Spheciospongia vesparium +++

Spirastella coccinea

Spongia obtusa

S. pertusa

Strongylacidon sp.

Spiratrella coccinea

Tedania ignis

Tethya crypta

Ulosa ruetzleri

U. funicularis

Verongula gigantea

V. rigida

Xetospongia muta

X. proxima

Especies de esponjas

38

42

30

11

CFAL

LZO

BAGU

BHONDA FRA

Géneros de esponjas

26

26

24

10

68
38

52

61

50

62

$+++=$ abundante $;++=$ común $;+=$ rara.

Localidades: $\mathrm{GRU}=$ Grumelandia; $\mathrm{PCOC}=$ Playa Coco; $\mathrm{CARRA}=$ Carraplanes; PLAN $=$ Punta Lanza; $\mathrm{CFAL}=\mathrm{Cabo}$ Falso; LZO = Lanza ZO; BAGUI = Bahía de Aguilas; BHONDA= Bahía Honda en Cabo Rojo; FRA = Los Frailes. 
